\title{
Edge regular graph products
}

\author{
Boštjan Frelih \\ UP IAM and UP FAMNIT \\ University of Primorska \\ Muzejski trg 2, 6000 Koper, Slovenia \\ bostjan.frelih@upr.si
}

\author{
Štefko Miklavič ${ }^{*}$ \\ UP IAM and UP FAMNIT \\ University of Primorska \\ Muzejski trg 2, 6000 Koper, Slovenia \\ stefko.miklavic@upr.si
}

Submitted: Oct 18, 2012; Accepted: Mar 11, 2013; Published: Mar 24, 2013

Mathematics Subject Classifications: 05C76

\begin{abstract}
A regular nonempty graph $\Gamma$ is called edge regular, whenever there exists a nonegative integer $\lambda_{\Gamma}$, such that any two adjacent vertices of $\Gamma$ have precisely $\lambda_{\Gamma}$ common neighbours. An edge regular graph $\Gamma$ with at least one pair of vertices at distance 2 is called amply regular, whenever there exists a nonegative integer $\mu_{\Gamma}$, such that any two vertices at distance 2 have precisely $\mu_{\Gamma}$ common neighbours. In this paper we classify edge regular graphs, which can be obtained as a strong product, or a lexicographic product, or a deleted lexicographic product, or a conormal product of two graphs. As a corollary we determine which of these graphs are amply regular. Keywords: edge regular graph; strong product; lexicographic

product; deleted lexicographic product; co-normal product
\end{abstract}

\section{Introductory remarks}

In this paper, the interplay between the concept of graph products and the concept of regularity of graphs is studied (see Section 2 for formal definitions). Both concepts recieved considerable attention in the mathematical literature. The interplay between these two concepts was studied, for example, in $[1,4,5]$, where distance-regular graphs, which can be obtained as a cartesian, tensor or strong product, were classified. In this paper we turn our attention to a larger class of graphs, namely edge regular and amply regular graphs. A regular nonempty graph $\Gamma$ is called edge regular, whenever there exists a nonegative integer $\lambda_{\Gamma}$, such that any two adjacent vertices of $\Gamma$ have precisely $\lambda_{\Gamma}$ common neighbours. An edge regular graph $\Gamma$ with at least one pair of vertices at distance 2 is

*Supported in part by "Agencija za raziskovalno dejavnost Republike Slovenije", research program P1-0285 and research project J1-4010. 
called amply regular, whenever there exists a nonegative integer $\mu_{\Gamma}$, such that any two vertices at distance 2 have precisely $\mu_{\Gamma}$ common neighbours. Note that distance-regular graphs are also amply regular (provided that their diameter is at least 2).

It is quite easy to see, that the cartesian or tensor product is edge regular if and only if both factors are. Therefore, in this paper we turn our attention to other graph products. We classify edge regular graphs, which can be obtained as a strong product, a lexicographic product, a deleted lexicographic product or a co-normal product of two graphs. As a corollary we determine which of these graphs are amply regular.

After some preliminaries in Section 2, we consider the case of strong product in Section 3 , the case of lexicographic product in Section 4, the case of deleted lexicographic product in Section 5, and the case of co-normal product in Section 6.

\section{Preliminaries}

In this section we review basic definitions and facts about edge regular graphs and graph products that we will need latter. Throughout this paper all graphs are assumed to be finite, undirected and without loops or multiple edges. For a graph $\Gamma$ we let $V(\Gamma)$, $E(\Gamma)$ and $\partial_{\Gamma}$ denote the vertex set, the edge set and the path length distance function, respectively. The diameter $\max \left\{\partial_{\Gamma}(x, y) \mid x, y \in V(\Gamma)\right\}$ of $\Gamma$ will be denoted by $D_{\Gamma}$.

For a positive integer $n$ we denote by $K_{n}$ ( $E_{n}$, respectively) the complete (empty, respectively) graph on $n$ vertices. We will denote a disjoint union of $t$ copies of $K_{n}$ by $t \times K_{n}$. A complete multipartite graph with $t$ partite sets each with $n$ vertices (that is, the complement of $t \times K_{n}$ ) will be denoted by $K_{t \times n}$.

For a vertex $x$ of a graph $\Gamma$, let $N_{\Gamma}(x)$ denote a set of vertices adjacent to $x$. If the number $\left|N_{\Gamma}(x)\right|$ is independent of the choice of a vertex $x \in \Gamma$, then we call this number the valency of $\Gamma$ and we denote it by $k_{\Gamma}$. In this case we say that $\Gamma$ is regular with valency $k_{\Gamma}$. Further degrees of regularity of $\Gamma$, namely edge regularity and amply regularity, can be defined as follows. Assume $\Gamma$ is nonempty. If $\left|N_{\Gamma}(x) \cap N_{\Gamma}(y)\right|$ is constant for every pair of adjacent vertices $x, y \in V(\Gamma)$, then we denote this common value with $\lambda_{\Gamma}$ and we say that the parameter $\lambda_{\Gamma}$ exists in $\Gamma$. Assume now that there is at least one pair of vertices of $\Gamma$ which are at distance 2 . If $\left|N_{\Gamma}(x) \cap N_{\Gamma}(y)\right|$ is constant for every pair of vertices $x, y \in V(\Gamma)$ with $\partial_{\Gamma}(x, y)=2$, then we denote this common value with $\mu_{\Gamma}$ and we say that the parameter $\mu_{\Gamma}$ exists in $\Gamma$. A regular nonempty graph $\Gamma$ is called edge regular if the parameter $\lambda_{\Gamma}$ exists in $\Gamma$. An edge regular graph $\Gamma$ is called amply regular if it contains a pair of vertices at distance 2 , and if the parameter $\mu_{\Gamma}$ exists in $\Gamma$. The following are well-known facts (see, for example, [2, page 3]).

Proposition 1. (i) Assume that $\Gamma$ is edge regular graph with $k_{\Gamma}=\lambda_{\Gamma}+1$. Then $\Gamma$ is $t \times K_{n}$ for some $t \geqslant 1$ and $n \geqslant 2$.

(ii) Assume that $\Gamma$ is a regular graph, such that any two nonadjacen vertices have no common neighbours. Then $\Gamma$ is $t \times K_{n}$ for some positive integers $t, n$. 
(iii) Assume that $\Gamma$ is a regular graph, such that any two nonadjacen vertices have exactly $k_{\Gamma}$ common neighbours. Then $\Gamma$ is $K_{t \times n}$ for some positive integers $t, n$.

We now define a strong product, a lexicographic product, a deleted lexicographic product, and a co-normal product of graphs $G$ and $H$. In all four cases, the vertex set of the product is $V(G) \times V(H)$. Pick vertices $\left(g_{1}, h_{1}\right)$ and $\left(g_{2}, h_{2}\right)$ in $V(G) \times V(H)$. In the strong product of $G$ and $H$, denoted by $G \otimes H,\left(g_{1}, h_{1}\right)$ and $\left(g_{2}, h_{2}\right)$ are adjacent if and only if $g_{1}=g_{2}$ and $h_{1}, h_{2}$ are adjacent in $H$, or $h_{1}=h_{2}$ and $g_{1}, g_{2}$ are adjacent in $G$, or $g_{1}, g_{2}$ are adjacent in $G$ and $h_{1}, h_{2}$ are adjacent in $H$. Note that strong product is commutative.

In the lexicographic product of $G$ and $H$, denoted by $G[H],\left(g_{1}, h_{1}\right)$ and $\left(g_{2}, h_{2}\right)$ are adjacent if and only if $g_{1}=g_{2}$ and $h_{1}, h_{2}$ are adjacent in $H$, or $g_{1}, g_{2}$ are adjacent in $G$.

Let $n=|V(H)|$. In the deleted lexicographic product of $G$ and $H$, denoted by $G[H]-$ $n G,\left(g_{1}, h_{1}\right)$ and $\left(g_{2}, h_{2}\right)$ are adjacent if and only if $g_{1}=g_{2}$ and $h_{1}, h_{2}$ are adjacent in $H$, or $g_{1}, g_{2}$ are adjacent in $G$ and $h_{1} \neq h_{2}$.

In the co-normal product of $G$ and $H,\left(g_{1}, h_{1}\right)$ and $\left(g_{2}, h_{2}\right)$ are adjacent if and only if $g_{1}, g_{2}$ are adjacent in $G$, or $h_{1}, h_{2}$ are adjacent in $H$. Note that co-normal product is commutative.

\section{Strong product}

Throughout this section let $G$ and $H$ be graphs and let $\Gamma=G \otimes H$ be the strong product of $G$ and $H$. We will classify graphs $G$ and $H$ for which $\Gamma$ is connected edge regular graph. Note that $\Gamma$ is connected if and only if $G$ and $H$ are connected. For the rest of this section we will assume that $\Gamma$ is connected (and thus also $G$ and $H$ are connected). To avoid trivialities we also assume that $|V(G)| \geqslant 2$ and $|V(H)| \geqslant 2$.

Lemma 2. $\Gamma$ is regular if and only if both $G$ and $H$ are regular. In this case the valency $k_{\Gamma}=k_{G}+k_{H}+k_{G} k_{H}$.

Proof. Assume $\Gamma$ is regular graph of valency $k_{\Gamma}$. For an arbitrary vertex $g \in V(G)$ take vertices $\left(g, h_{1}\right),\left(g, h_{2}\right) \in V(\Gamma)$. By the definition of $\Gamma$ we have

$$
k_{\Gamma}=\left|N_{\Gamma}\left(\left(g, h_{1}\right)\right)\right|=\left|N_{G}(g)\right|+\left|N_{H}\left(h_{1}\right)\right|+\left|N_{G}(g)\right|\left|N_{H}\left(h_{1}\right)\right|,
$$

and

$$
k_{\Gamma}=\left|N_{\Gamma}\left(\left(g, h_{2}\right)\right)\right|=\left|N_{G}(g)\right|+\left|N_{H}\left(h_{2}\right)\right|+\left|N_{G}(g)\right|\left|N_{H}\left(h_{2}\right)\right| .
$$

It follows that $\left|N_{H}\left(h_{1}\right)\right|=\left|N_{H}\left(h_{2}\right)\right|$, so $H$ is regular. The proof that $G$ is regular is analogous.

Conversely, if both $G$ and $H$ are regular, then $\Gamma$ is obviously a regular graph, as

$$
\left|N_{\Gamma}((g, h))\right|=\left|N_{G}(g)\right|+\left|N_{H}(h)\right|+\left|N_{G}(g)\right|\left|N_{H}(h)\right|=k_{G}+k_{H}+k_{G} k_{H}
$$

for every $(g, h) \in V(\Gamma)$. 
Lemma 3. Assume that $\Gamma$ is edge regular. Then $G$ and $H$ are also edge regular.

Proof. Recall that $G$ and $H$ are regular by Lemma 2. Pick adjacent vertices $g_{1}, g_{2}$ of $G$. For an arbitrary vertex $h \in V(H)$ consider $u=\left(g_{1}, h\right), v=\left(g_{2}, h\right) \in V(\Gamma)$. Note that, by the definition of $\Gamma, \partial_{\Gamma}(u, v)=1$. We now count the number of common neighbours of $u$ and $v$. Note that we have

$$
\begin{aligned}
N_{\Gamma}(u) \cap N_{\Gamma}(v)= & \left\{(g, h) \mid g \in N_{G}\left(g_{1}\right) \cap N_{G}\left(g_{2}\right)\right\} \cup \\
& \left\{\left(g_{1}, h_{1}\right),\left(g_{2}, h_{2}\right) \mid h_{1}, h_{2} \in N_{H}(h)\right\} \cup \\
& \left\{\left(g_{3}, h_{3}\right) \mid g_{3} \in N_{G}\left(g_{1}\right) \cap N_{G}\left(g_{2}\right), h_{3} \in N_{H}(h)\right\} .
\end{aligned}
$$

Therefore

$$
\left|N_{\Gamma}(u) \cap N_{\Gamma}(v)\right|=\left|N_{G}\left(g_{1}\right) \cap N_{G}\left(g_{2}\right)\right|+2 k_{H}+k_{H}\left|N_{G}\left(g_{1}\right) \cap N_{G}\left(g_{2}\right)\right| .
$$

Since $\lambda_{\Gamma}=\left|N_{\Gamma}(u) \cap N_{\Gamma}(v)\right|$, it follows that

$$
\left|N_{G}\left(g_{1}\right) \cap N_{G}\left(g_{2}\right)\right|=\frac{\lambda_{\Gamma}-2 k_{H}}{k_{H}+1} .
$$

Therefore, the number of common neighbours of two adjacent vertices of $G$ is independent of the choice of these vertices, and thus $\lambda_{G}$ exists. The commutativity of the strong product implies the proof for $H$.

Corollary 4. If $\Gamma$ is edge regular, then

$$
\lambda_{\Gamma}=2 k_{H}+\left(k_{H}+1\right) \lambda_{G}=2 k_{G}+\left(k_{G}+1\right) \lambda_{H}=\lambda_{H} \lambda_{G}+2 \lambda_{G}+2 \lambda_{H}+2 .
$$

Proof. Observe thet by Lemma 2 and Lemma 3, $G$ and $H$ are both edge regular, so $\lambda_{G}$ and $\lambda_{H}$ exist. The first equality now follows from (1). The second equality is obtained by reversing the roles of $G$ and $H$. To prove the third equality, pick adjacent vertices $g_{1}, g_{2}$ of $G$ and adjacent vertices $h_{1}, h_{2}$ of $H$. Consider vertices $u=\left(g_{1}, h_{1}\right), v=\left(g_{2}, h_{2}\right) \in V(\Gamma)$. Note that, by the definition of $\Gamma, \partial_{\Gamma}(u, v)=1$. We now count the number of common neighbours of $u$ and $v$.

Two of them are $\left(g_{1}, h_{2}\right)$ and $\left(g_{2}, h_{1}\right)$. There is $2 \lambda_{G}$ common neighbours whose second coordinate is $h_{1}$ (and first coordinate is not $g_{2}$ ) or $h_{2}$ (and first coordinate is not $g_{1}$ ), namely vertices of the form $\left(x, h_{1}\right)$ and $\left(x, h_{2}\right)$, where $x \in N_{G}\left(g_{1}\right) \cap N_{G}\left(g_{2}\right)$. Similarly, there is $2 \lambda_{H}$ common neighbours whose first coordinate is $g_{1}$ (and second coordinate is not $h_{2}$ ) or $g_{2}$ (and second coordinate is not $h_{1}$ ). Finaly, there is $\lambda_{G} \lambda_{H}$ common neighbours whose first coordinate is not $g_{1}$ or $g_{2}$, and whose second coordinate is not $h_{1}$ or $h_{2}$. These vertices are vertices of the form $(x, y)$, where $x \in N_{G}\left(g_{1}\right) \cap N_{G}\left(g_{2}\right)$ and $y \in N_{H}\left(h_{1}\right) \cap N_{H}\left(h_{2}\right)$. The result follows.

Theorem 5. $\Gamma=G \otimes H$ is edge regular if and only if $G=K_{n}$ and $H=K_{m}$ for some $n, m \geqslant 2$. 
Proof. Assume that $\Gamma$ is edge regular. By Corollary 4 we have $2 k_{G}+\left(k_{G}+1\right) \lambda_{H}=$ $\lambda_{H} \lambda_{G}+2 \lambda_{G}+2 \lambda_{H}+2$, implying that

$$
\left(k_{G}-\lambda_{G}\right)\left(\lambda_{H}+2\right)=\lambda_{H}+2 .
$$

It follows that $k_{G}=\lambda_{G}+1$. By Proposition 1(i) and since $G$ is connected, $G=K_{n}$ for some $n \geqslant 2$. Similarly we prove that $H=K_{m}$ for some $m \geqslant 2$.

Assume now that $G=K_{n}$ and $H=K_{m}$ for some $n, m \geqslant 2$. Note that in this case $\Gamma$ is $K_{m n}$, which is clearly edge regular.

Corollary 6. $\Gamma=G \otimes H$ is never amply regular.

Proof. Assume that $\Gamma$ is amply regular. Since in this case $\Gamma$ is also edge regular, $G=K_{n}$ and $H=K_{m}$ for some $n, m \geqslant 2$ by Theorem 5 . But then $\Gamma$ is $K_{m n}$, which is not amply regular (since no two vertices are at distance 2 ), a contradiction.

\section{Lexicographic product}

Throughout this section let $G$ and $H$ be graphs and let $\Gamma=G[H]$ be the lexicographic product of $G$ and $H$. We will classify graphs $G$ and $H$ for which $\Gamma$ is connected edge regular graph. Note that $\Gamma$ is connected if and only if $G$ is connected. For the rest of this section we will assume that $\Gamma$ is connected (and thus $G$ is also connected). To avoid trivialities we also assume that $|V(G)| \geqslant 2$ and $|V(H)| \geqslant 2$.

Lemma 7. $\Gamma$ is regular if and only if both $G$ and $H$ are regular. In this case the valency $k_{\Gamma}=k_{H}+k_{G}|V(H)|$.

Proof. Assume $\Gamma$ is regular graph of valency $k_{\Gamma}$. For an arbitrary vertex $g \in V(G)$ take vertices $\left(g, h_{1}\right),\left(g, h_{2}\right) \in V(\Gamma)$. By the definition of $\Gamma$ we have

$$
k_{\Gamma}=\left|N_{\Gamma}\left(\left(g, h_{1}\right)\right)\right|=\left|N_{H}\left(h_{1}\right)\right|+\left|N_{G}(g)\right||V(H)|,
$$

and

$$
k_{\Gamma}=\left|N_{\Gamma}\left(\left(g, h_{2}\right)\right)\right|=\left|N_{H}\left(h_{2}\right)\right|+\left|N_{G}(g)\right||V(H)| .
$$

It follows that $\left|N_{H}\left(h_{1}\right)\right|=\left|N_{H}\left(h_{2}\right)\right|$, so $H$ is regular. Now we also have that

$$
k_{\Gamma}=\left|N_{\Gamma}\left(\left(g, h_{1}\right)\right)\right|=\left|N_{G}(g)\right||V(H)|+k_{H} .
$$

It follows that $\left|N_{G}(g)\right|=\left(k_{\Gamma}-k_{H}\right) /|V(H)|$, so $G$ is regular.

Conversely, if both $G$ and $H$ are regular, then $\Gamma$ is obviously regular, as

$$
\left|N_{\Gamma}((g, h))\right|=\left|N_{H}(h)\right|+\left|N_{G}(g)\right||V(H)|=k_{H}+k_{G}|V(H)|
$$

for every $(g, h) \in V(\Gamma)$.

Lemma 8. Assume that $\Gamma$ is edge regular. Then the following (i), (ii) hold. 
(i) $G$ is edge regular.

(ii) Either $H=E_{m}$ for some positive integer $m$, or $H$ is edge regular.

Proof. Recall that $G$ and $H$ are regular by Lemma 7.

(i) Pick a pair of adjacent vertices $g_{1}, g_{2}$ of $G$. For an arbitrary vertex $h \in V(H)$ consider $u=\left(g_{1}, h\right), v=\left(g_{2}, h\right) \in V(\Gamma)$. Note that, by the definition of $\Gamma, \partial_{\Gamma}(u, v)=1$. We now count the number of common neighbours of $u$ and $v$. Observe that

$$
\begin{aligned}
N_{\Gamma}(u) \cap N_{\Gamma}(v)= & \left\{\left(g_{1}, h^{\prime}\right),\left(g_{2}, h^{\prime}\right) \mid h^{\prime} \in N_{H}(h)\right\} \cup \\
& \left\{\left(g^{\prime}, h^{\prime}\right) \mid g^{\prime} \in N_{G}\left(g_{1}\right) \cap N_{G}\left(g_{2}\right), h^{\prime} \in V(H)\right\} .
\end{aligned}
$$

Therefore

$$
\left|N_{\Gamma}(u) \cap N_{\Gamma}(v)\right|=2\left|N_{H}(h)\right|+\left|N_{G}\left(g_{1}\right) \cap N_{G}\left(g_{2}\right)\right||V(H)| .
$$

Since $\lambda_{\Gamma}=\left|N_{\Gamma}(u) \cap N_{\Gamma}(v)\right|$, it follows that

$$
\left|N_{G}\left(g_{1}\right) \cap N_{G}\left(g_{2}\right)\right|=\frac{\lambda_{\Gamma}-2 k_{H}}{|V(H)|} .
$$

Therefore, the number of common neighbours of two adjacent vertices of $G$ is independent of the choice of these vertices, and thus $\lambda_{G}$ exists. The result follows.

(ii) Assume that $H$ is nonempty. Pick a pair of adjacent vertices $h_{1}, h_{2}$ of $H$. For an arbitrary vertex $g \in V(G)$ consider vertices $u=\left(g, h_{1}\right), v=\left(g, h_{2}\right) \in V(\Gamma)$. Note that, by the definition of $\Gamma, \partial_{\Gamma}(u, v)=1$. We now count the number of common neighbours of $u$ and $v$ in $\Gamma$. Observe that

$$
N_{\Gamma}(u) \cap N_{\Gamma}(v)=\left\{(g, h) \mid h \in N_{H}\left(h_{1}\right) \cap N_{H}\left(h_{2}\right)\right\} \cup\left\{\left(g^{\prime}, h^{\prime}\right) \mid g^{\prime} \in N_{G}(g), h^{\prime} \in V(H)\right\} .
$$

Therefore

$$
\left|N_{\Gamma}(u) \cap N_{\Gamma}(v)\right|=\left|N_{H}\left(h_{1}\right) \cap N_{H}\left(h_{2}\right)\right|+\left|N_{G}(g)\right||V(H)| .
$$

Since $\lambda_{\Gamma}=\left|N_{\Gamma}(u) \cap N_{\Gamma}(v)\right|$, it follows that

$$
\left|N_{H}\left(h_{1}\right) \cap N_{H}\left(h_{2}\right)\right|=\lambda_{\Gamma}-k_{G}|V(H)| .
$$

Therefore, the number of common neighbours of two adjacent vertices in $H$ is independent of the choice of these two vertices, and thus $\lambda_{H}$ exists. The result follows.

Corollary 9. If $\Gamma$ is edge regular and $H$ is nonempty, then

$$
\lambda_{\Gamma}=2 k_{H}+\lambda_{G}|V(H)|=k_{G}|V(H)|+\lambda_{H} .
$$

Proof. Observe that by Lemma 7, $G$ and $H$ are both regular. By Lemma $8, \lambda_{G}$ and $\lambda_{H}$ exist. The result now follows from equalities (2) and (3).

Theorem 10. $\Gamma=G[H]$ is edge regular if and only if one of the following holds: 
(i) $H=E_{m}$ for some $m \geqslant 2$ and $G$ is edge regular.

(ii) $G=K_{n}$ and $H=K_{t \times m}$ for some $n, t \geqslant 2$ and $m \geqslant 1$.

Proof. Assume that $\Gamma$ is edge regular. By Lemma 7 and Lemma 8, $G$ is edge regular and $H$ is regular. If $H$ is an empty graph $E_{m}$ for some $m \geqslant 2$, then we are done. Assume now that $H$ is nonempty. By Corollary $9, k_{G}|V(H)|+\lambda_{H}=2 k_{H}+\lambda_{G}|V(H)|$. It follows that

$$
|V(H)|\left(k_{G}-\lambda_{G}\right)=2 k_{H}-\lambda_{H} .
$$

Since obviously $\lambda_{G}<k_{G}$ and $k_{H}<|V(H)|$ we have that $k_{G}-\lambda_{G}=1$ and that $2 k_{H}-$ $\lambda_{H}=|V(H)|$. By Proposition 1(i) and since $G$ is connected, $G$ is a complete graph. Furthermore, note that in the complement of $H$, every two nonadjacent vertices have exactly $|V(H)|-2 k_{H}+\lambda_{H}=0$ common neighbours. Since the complement of $H$ is also regular, Proposition 1(ii) implies that the complement of $H$ is isomorphic to $t \times K_{m}$ for some $t, m \geqslant 1$. It follows that $H=K_{t \times m}$ for some $t, m \geqslant 1$. Since $H$ is nonempty, we also have that $t \geqslant 2$.

Assume now that (i) or (ii) of the Theorem holds. Then $\Gamma$ is regular by Lemma 7 . It is now an easy exercise to show that if (i) holds, then $\lambda_{\Gamma}=\lambda_{G}|V(H)|$, and that if (ii) holds, then $\Gamma=K_{t n \times m}$, which is clearly edge regular with $\lambda_{\Gamma}=t n m-2 m$.

Corollary 11. $\Gamma=G[H]$ is amply regular if and only if one of the following holds:

(i) $H=E_{m}$ for some $m \geqslant 2$ and $G=K_{t \times n}$ for some $t \geqslant 2, n \geqslant 1$.

(ii) $G=K_{n}$ and $H=K_{t \times m}$ for some $n, m, t \geqslant 2$.

Proof. Assume that $\Gamma$ is amply regular. Let first consider case (i) of Theorem 10, that is the case when $H=E_{m}$ for some $m \geqslant 2$ and $G$ is edge regular. If $G$ is a complete graph, then (i) above holds. Therefore assume that $G$ is not a complete graph. Pick $g \in V(G)$ and $h_{1}, h_{2} \in V(H)$. Abbreviate $u=\left(g, h_{1}\right), v=\left(g, h_{2}\right)$ and note that $\partial_{\Gamma}(u, v)=2$. It is also clear that

$$
\left|N_{\Gamma}(u) \cap N_{\Gamma}(v)\right|=k_{G}|V(H)| .
$$

Next pick arbitrary $g_{1}, g_{2} \in V(G)$ with $\partial_{G}\left(g_{1}, g_{2}\right)=2$, and arbitrary $h \in V(H)$. Abbreviate $w=\left(g_{1}, h\right), z=\left(g_{2}, h\right)$ and note that $\partial_{\Gamma}(w, z)=2$. In this case we have

$$
\left|N_{\Gamma}(w) \cap N_{\Gamma}(z)\right|=\left|N_{G}\left(g_{1}\right) \cap N_{G}\left(g_{2}\right)\right||V(H)| .
$$

If $\Gamma$ is amply regular, then we clearly have

$$
k_{G}|V(H)|=\left|N_{G}\left(g_{1}\right) \cap N_{G}\left(g_{2}\right)\right||V(H)|,
$$

which implies $k_{G}=\left|N_{G}\left(g_{1}\right) \cap N_{G}\left(g_{2}\right)\right|$. Therefore, $\mu_{G}$ exists and is equal to $k_{G}$. Note that this implies that for $x, y \in V(G)$ we have $\partial_{G}(x, y) \leqslant 2$ (since in $G$ there is no induced path of length 3), and so by Proposition 1(iii), $G$ is $K_{t \times n}$ for some positive integers $t, n$. As $G$ is connected, we clearly have that $t \geqslant 2$.

Let us now consider case (ii) of Theorem 10, that is $G=K_{n}$ and $H=K_{t \times m}$ for some $n, t \geqslant 2$ and $m \geqslant 1$. Since $\Gamma$ is not a complete graph, we have $m \geqslant 2$.

Conversely, if (i) above holds, then $\Gamma=K_{t \times m n}$, and if (ii) above holds, then $\Gamma=K_{t n \times m}$. In both cases $\Gamma$ is an amply graph. 


\section{Deleted lexicographic product}

Throughout this section let $G$ and $H$ be graphs and let $\Gamma=G[H]-n G$ be the deleted lexicographic product of $G$ and $H$, where $n=|V(H)|$. We will classify graphs $G$ and $H$ for which $\Gamma$ is connected edge regular graph. To avoid trivialities we assume that $|V(G)| \geqslant 2$ and $|V(H)| \geqslant 2$.

Lemma 12. (i) If $H=E_{2}$, then $\Gamma$ is connected if and only if $G$ is connected and nonbipartite.

(ii) If $H \neq E_{2}$, then $\Gamma$ is connected if and only if $G$ is connected.

Proof. (i) Note that, if $H=E_{2}$, then $\Gamma$ is isomorphic to the tensor product $G \times K_{2}$ (for the definition of tensor product see [3, Section 5.3]). The result now follows from [3, Theorem 5.29].

(ii) Assume now that $H \neq E_{2}$. If $G$ is not connected, then $\Gamma$ is obviously not connected. Conversely, assume that $G$ is connected. Pick two arbitrary vertices $\left(g_{1}, h_{1}\right),\left(g_{2}, h_{2}\right)$ of $\Gamma$. We claim that there exist a path in $\Gamma$ between $\left(g_{1}, h_{1}\right),\left(g_{2}, h_{2}\right)$. Since $G$ is connected, there exists a path between $g_{1}$ and $g_{2}$ in $G$. It follows that there exists a path between $\left(g_{1}, h_{1}\right)$ and $\left(g_{2}, h\right)$ for some vertex $h \in H$. If $h=h_{2}$ or if $h, h_{2}$ are adjacent in $H$, then we are done. Now if $h \neq h_{2}$ and $h, h_{2}$ are not adjacent in $H$, then $H$ is not $K_{2}$. Since $H$ is also not $E_{2}$, this shows that $|V(H)| \geqslant 3$. Now pick a neighbour $g$ of $g_{2}$ in $G$ and $h^{\prime} \in V(H) \backslash\left\{h_{2}, h\right\}$. Note that $\left(g, h^{\prime}\right)$ is a common neighbour of $\left(g_{2}, h_{2}\right)$ and $\left(g_{2}, h\right)$ in $\Gamma$. This verifies the claim and thus $\Gamma$ is connected.

Lemma 13. $\Gamma$ is regular if and only if both $H$ and $G$ are regular. In this case the valency $k_{\Gamma}=k_{H}+k_{G}(|V(H)|-1)$.

Proof. Assume $\Gamma$ is regular graph of valency $k_{\Gamma}$. For an arbitrary vertex $g \in V(G)$ take vertices $\left(g, h_{1}\right),\left(g, h_{2}\right) \in V(\Gamma)$. By the definition of $\Gamma$ we have

$$
k_{\Gamma}=\left|N_{\Gamma}\left(\left(g, h_{1}\right)\right)\right|=\left|N_{H}\left(h_{1}\right)\right|+\left|N_{G}(g)\right|(|V(H)|-1)
$$

and

$$
k_{\Gamma}=\left|N_{\Gamma}\left(\left(g, h_{2}\right)\right)\right|=\left|N_{H}\left(h_{2}\right)\right|+\left|N_{G}(g)\right|(|V(H)|-1) .
$$

It follows that $\left|N_{H}\left(h_{1}\right)\right|=\left|N_{H}\left(h_{2}\right)\right|$, so $H$ is regular. We now also have

$$
k_{\Gamma}=\left|N_{\Gamma}\left(\left(g, h_{1}\right)\right)\right|=k_{H}+\left|N_{G}(g)\right|(|V(H)|-1) .
$$

It follows that $\left|N_{G}(g)\right|=\left(k_{\Gamma}-k_{H}\right) /(|V(H)|-1)$, so $G$ is regular.

Conversely, if both $G$ and $H$ are regular, then $\Gamma$ is obviously a regular graph, as

$$
\left|N_{\Gamma}((g, h))\right|=\left|N_{H}(h)\right|+\left|N_{G}(g)\right|(|V(H)|-1)=k_{H}+k_{G}(|V(H)|-1)
$$

for every $(g, h) \in V(\Gamma)$.

For the rest of this section we will assume that $\Gamma$ is connected (and thus $G$ is also connected). 


\subsection{The case $|V(H)|=2$}

Recall that $\Gamma=G[H]-n G$ is the deleted lexicographic product of $G$ and $H$, where $n=|V(H)|$. In this subsection we consider the case $|V(H)|=2$.

Theorem 14. Assume that $|V(H)|=2$. Then $\Gamma$ is edge regular if and only if one of the the following (i), (ii) holds.

(i) $H=E_{2}$ and $G$ is regular and nonbipartite.

(ii) $H=K_{2}$ and $G$ is regular.

Proof. Observe that since $|V(H)|=2, \Gamma$ is bipartite. Therefore, if $\Gamma$ is edge regular, then $\lambda_{\Gamma}=0$.

Assume $\Gamma$ is edge regular. In particular, $\Gamma$ is regular and connected. If $H=E_{2}$, then $G$ is nonbipartite by Lemma $12(\mathrm{i})$ and regular by Lemma 13 . If $H=K_{2}$, then $G$ is regular by Lemma 13.

Conversely, if (i) or (ii) holds, then $\Gamma$ is regular by Lemma 13. Since $\Gamma$ is bipartite, we have $\lambda_{\Gamma}=0$.

Let us now determine in which cases of Theorem 14 the graph $\Gamma$ is amply regular. Assume first that $H=E_{2}$. As already mentioned in the proof of Lemma 12, in this case $\Gamma$ is isomorphic to the tensor product $G \times K_{2}$, and it is also called a bipartite double of $G$, see [2, Section 1.11]. Recall also that $\lambda_{\Gamma}=0$. By [2, Theorem 1.11.1 (ii)], $\Gamma$ is amply regular if and only if $G$ is regular nonbipartite graph, in which any two distinct vertices have either 0 or $\mu_{\Gamma}$ common neighbours. Let us now turn our attention to the case when $H=K_{2}$.

Lemma 15. Assume that $H=K_{2}$ and that $\Gamma$ is amply regular. Then the following(i)-(iii) hold.

(i) $G$ is edge regular.

(ii) Either $G$ is a complete graph or $G$ is amply regular.

(iii) If $G$ is not a complete graph, then $\lambda_{G}+2=\mu_{G}=\mu_{\Gamma}$.

Proof. Set $V(H)=\left\{h_{1}, h_{2}\right\}$.

(i) Pick adjacent vertices $g_{1}, g_{2}$ of $G$. Consider vertices $u=\left(g_{1}, h_{1}\right)$ and $v=\left(g_{2}, h_{1}\right)$ of $\Gamma$. Note that $\partial_{\Gamma}(u, v)=2$. Let us count the number of common neighbours of $u$ and $v$. Note that $z=(g, h)$ is a common neighbour of $u$ and $v$ if and only if $h=h_{2}$, and either $g \in\left\{g_{1}, g_{2}\right\}$ or $g$ is a common neighbor of $g_{1}, g_{2}$ in $G$. Therefore,

$$
\mu_{\Gamma}=\left|N_{\Gamma}(u) \cap N_{\Gamma}(v)\right|=2+\left|N_{G}\left(g_{1}\right) \cap N_{G}\left(g_{2}\right)\right| .
$$

It follows that the number of common neighbours of two adjacent vertices in $G$ is independent of the choice of these two vertices, and thus $\lambda_{G}$ exists. 
(ii) Assume that $G$ is not a complete graph, and pick vertices $g_{1}, g_{2}$ of $G$ such that $\partial_{G}\left(g_{1}, g_{2}\right)=2$. Consider vertices $u=\left(g_{1}, h_{1}\right)$ and $v=\left(g_{2}, h_{1}\right)$ of $\Gamma$. Note that $\partial_{\Gamma}(u, v)=2$. Let us count the number of common neighbours of $u$ and $v$. Note that $z=(g, h)$ is a common neighbour of $u$ and $v$ if and only if $h=h_{2}$ and $g$ is a common neighbor of $g_{1}, g_{2}$ in $G$. Therefore,

$$
\mu_{\Gamma}=\left|N_{\Gamma}(u) \cap N_{\Gamma}(v)\right|=\left|N_{G}\left(g_{1}\right) \cap N_{G}\left(g_{2}\right)\right| .
$$

It follows that the number of common neighbours of two vertices of $G$ which are at distance 2 is independent of the choice of these two vertices, and thus $\mu_{G}$ exists.

(iii) This follows from (4) and (5).

Theorem 16. Assume that $H=K_{2}$. Then $\Gamma=G[H]-n G$ is amply regular if and only if either $G$ is a complete graph, or $G$ is an amply regular graph with $\lambda_{G}+2=\mu_{G}$.

Proof. Assume first that $\Gamma$ is an amply regular graph and that $G$ is not a complete graph. Then $G$ is amply regular with $\lambda_{G}+2=\mu_{G}$ by Lemma 15(iii).

Conversly, assume first that $G$ is a complete graph $K_{m}$. Then $\Gamma$ is isomorphic to the complete bipartite graph $K_{m, m}$, which is clearly amply regular. Assume now that $G$ is an amply regular graph with $\lambda_{G}+2=\mu_{G}$. Since $\Gamma$ is bipartite, $\Gamma$ is edge regular with $\lambda_{\Gamma}=0$. Now pick vertices $u=\left(g_{1}, h_{1}\right), v=\left(g_{2}, h_{2}\right)$ of $\Gamma$ such that $\partial_{\Gamma}(u, v)=2$. Note that we have two possibilities:

(i) $h_{1}=h_{2}$ and $g_{1}, g_{2}$ are adjacent in $G$. In this case $u$ and $v$ have $2+\lambda_{G}$ common neighbours.

(ii) $h_{1}=h_{2}$ and $\partial_{G}\left(g_{1}, g_{2}\right)=2$. In this case $u$ and $v$ have $\mu_{G}$ common neighbours.

As $\lambda_{G}+2=\mu_{G}$, the number of common neighbours of $u$ and $v$ is independent on the choice of these two vertices. This shows that $\mu_{\Gamma}$ exists and so $\Gamma$ is amply regular.

\subsection{Case $|V(H)| \geqslant 3$}

Let us now turn our attention to the case $|V(H)| \geqslant 3$.

Lemma 17. If $\Gamma$ is edge regular, then either $H=E_{n}$ or $H=K_{n}$ for some $n \geqslant 3$.

Proof. Assume that $\Gamma$ is edge regular and that $H \neq E_{n}, H \neq K_{n}$. Then there exist (distinct) vertices $h_{1}, h_{2}, h_{3} \in V(H)$, such that $\partial_{H}\left(h_{1}, h_{2}\right)=1$ and $\partial_{H}\left(h_{1}, h_{3}\right) \neq 1$. Pick adjacent vertices $g_{1}, g_{2} \in V(G)$ and consider vertices $u=\left(g_{1}, h_{1}\right), v=\left(g_{2}, h_{2}\right)$ and $z=\left(g_{2}, h_{3}\right) \in V(\Gamma)$. Note that, by the definition of $\Gamma, \partial_{\Gamma}(u, v)=1$ and $\partial_{\Gamma}(u, z)=1$. Now count the number of common neighbours of adjacent vertices $u, v$ and $u, z$ of $\Gamma$. Observe that we have

$$
\begin{aligned}
N_{\Gamma}(u) \cap N_{\Gamma}(v)= & \left\{\left(g_{1}, h\right) \mid h \in N_{H}\left(h_{1}\right) \backslash\left\{h_{2}\right\}\right\} \cup \\
& \left\{\left(g_{2}, h\right) \mid h \in N_{H}\left(h_{2}\right) \backslash\left\{h_{1}\right\}\right\} \cup \\
& \left\{(g, h) \mid g \in N_{G}\left(g_{1}\right) \cap N_{G}\left(g_{2}\right), h \in V(H) \backslash\left\{h_{1}, h_{2}\right\}\right\}
\end{aligned}
$$

THE ELECTRONic Journal OF COMBinatorics 20(1) (2013), \#P62 
and

$$
\begin{aligned}
N_{\Gamma}(u) \cap N_{\Gamma}(z)= & \left\{\left(g_{1}, h\right) \mid h \in N_{H}\left(h_{1}\right)\right\} \cup \\
& \left\{\left(g_{2}, h\right) \mid h \in N_{H}\left(h_{3}\right)\right\} \cup \\
& \left\{(g, h) \mid g \in N_{G}\left(g_{1}\right) \cap N_{G}\left(g_{2}\right), h \in V(H) \backslash\left\{h_{1}, h_{3}\right\}\right\} .
\end{aligned}
$$

Therefore

$$
\left|N_{\Gamma}(u) \cap N_{\Gamma}(v)\right|=2\left(k_{H}-1\right)+\left|N_{G}\left(g_{1}\right) \cap N_{G}\left(g_{2}\right)\right|(|V(H)|-2),
$$

and

$$
\left|N_{\Gamma}(u) \cap N_{\Gamma}(z)\right|=2 k_{H}+\left|N_{G}\left(g_{1}\right) \cap N_{G}\left(g_{2}\right)\right|(|V(H)|-2) .
$$

Obviously

$$
\left|N_{\Gamma}(u) \cap N_{\Gamma}(v)\right| \neq\left|N_{\Gamma}(w) \cap N_{\Gamma}(z)\right|,
$$

a contradiction with assumption that $\lambda_{\Gamma}$ exists. It follows that either $H=E_{n}$ or $H=$ $K_{n}$.

Lemma 18. Assume that $\Gamma$ is edge regular. Then $G$ is edge regular.

Proof. Recall that $G$ is regular by Lemma 13. Recall also that either $H=E_{n}$ or $H=K_{n}$ by Lemma 17 , where $n=|V(H)|$. Pick a pair of adjacent vertices $g_{1}, g_{2}$ of $G$. For arbitrary vertices $h_{1}, h_{2} \in V(H)\left(h_{1} \neq h_{2}\right)$ consider $u=\left(g_{1}, h_{1}\right), v=\left(g_{2}, h_{2}\right) \in V(\Gamma)$. Note that, by the definition of $\Gamma, \partial_{\Gamma}(u, v)=1$. We now count the number of common neighbours of $u$ and $v$. Observe that

$$
\begin{aligned}
N_{\Gamma}(u) \cap N_{\Gamma}(v)= & \left\{\left(g_{1}, h\right) \mid h \in N_{H}\left(h_{1}\right) \backslash\left\{h_{2}\right\}\right\} \cup \\
& \left\{\left(g_{2}, h\right) \mid h \in N_{H}\left(h_{2}\right) \backslash\left\{h_{1}\right\}\right\} \cup \\
& \left\{(g, h) \mid g \in N_{G}\left(g_{1}\right) \cap N_{G}\left(g_{2}\right), h \in V(H) \backslash\left\{h_{1}, h_{2}\right\}\right\} .
\end{aligned}
$$

Therefore

$$
\left|N_{\Gamma}(u) \cap N_{\Gamma}(v)\right|=2 t+\left|N_{G}\left(g_{1}\right) \cap N_{G}\left(g_{2}\right)\right|(n-2),
$$

where $t=0$ if $H=E_{n}$ and $t=n-2$ if $H=K_{n}$. Since $\lambda_{\Gamma}=\left|N_{\Gamma}(u) \cap N_{\Gamma}(v)\right|$, it follows that

$$
\left|N_{G}\left(g_{1}\right) \cap N_{G}\left(g_{2}\right)\right|=\frac{\lambda_{\Gamma}-2 t}{n-2} .
$$

Therefore, the number of common neighbours of two adjacent vertices of $G$ is independent of the choice of these vertices, and thus $\lambda_{G}$ exists.

Corollary 19. If $\Gamma$ is edge regular and $H=K_{n}$ for some $n \geqslant 3$, then

$$
\lambda_{\Gamma}=\left(\lambda_{G}+2\right)(n-2)=\left(k_{G}+1\right)(n-2) .
$$


Proof. Observe that $\lambda_{\Gamma}=\left(\lambda_{G}+2\right)(n-2)$ follows from (6). To prove that $\lambda_{\Gamma}=\left(k_{G}+\right.$ $1)(n-2)$, pick $g \in V(G)$ and $h_{1}, h_{2} \in V(H), h_{1} \neq h_{2}$. Consider vertices $u=\left(g, h_{1}\right)$ and $v=\left(g, h_{2}\right)$. Note that $\partial_{\Gamma}(u, v)=1$. Let us now count the number of common neighbours of $u$ and $v$. Obviously we have

$$
N_{\Gamma}(u) \cap N_{\Gamma}(v)=\left\{\left(g_{1}, h\right) \mid g_{1} \in N_{G}(g) \cup\{g\}, h \in V(H) \backslash\left\{h_{1}, h_{2}\right\}\right\} .
$$

It follows

$$
\lambda_{\Gamma}=\left|N_{\Gamma}(u) \cap N_{\Gamma}(v)\right|=\left(k_{G}+1\right)(n-2) .
$$

Theorem 20. $\Gamma=G[H]-n G$ is edge regular if and only if one of the following (i), (ii) holds:

(i) $H=E_{n}$ for some $n \geqslant 3$ and $G$ is edge regular.

(ii) $H=K_{n}$ for some $n \geqslant 3$ and $G=K_{m}$ for some $m \geqslant 2$.

Proof. Assume that $\Gamma$ is edge regular. Recall that $H=E_{n}$ or $H=K_{n}$ by Lemma 17 . Furthermore, $G$ is edge regular by Lemma 18. Assume now that $H=K_{n}$ for some $n \geqslant 3$. By Corollary 19 we have $\lambda_{G}+1=k_{G}$. By Proposition 1(i) and since we suppose that $G$ is connected, we have that $G=K_{m}$ for some $m \geqslant 2$.

Conversly, assume first that $H=E_{n}$ for some $n \geqslant 3$ and that $G$ is edge regular. Pick adjacent vertices $u=\left(g_{1}, h_{1}\right)$ and $v=\left(g_{2}, h_{2}\right)$ of $\Gamma$. Note that $g_{1}, g_{2}$ are adjacent in $G$ and that $h_{1} \neq h_{2}$. Moreover,

$$
N_{\Gamma}(u) \cap N_{\Gamma}(v)=\left\{(g, h) \mid g \in N_{G}\left(g_{1}\right) \cap N_{G}\left(g_{2}\right), h \in V(H) \backslash\left\{h_{1}, h_{2}\right\}\right\} .
$$

Therefore

$$
\left|N_{\Gamma}(u) \cap N_{\Gamma}(v)\right|=\lambda_{G}(n-2),
$$

so $\Gamma$ is edge regular.

Assume now that $H=K_{n}$ for some $n \geqslant 3$ and $G=K_{m}$ for some $m \geqslant 2$. Observe that $\Gamma$ is isomorphic to $K_{n \times m}$, which is clearly edge regular.

Lemma 21. If $\Gamma$ is amply regular, then $G=K_{m}$ for some $m \geqslant 2$.

Proof. Assume $\Gamma$ is amply regular and that $G \neq K_{m}$. By Theorem $20, H=E_{n}(n \geqslant 3)$, where $n=|V(H)|$. Pick $g_{1}, g_{2} \in V(G)$ such that $\partial_{G}\left(g_{1}, g_{2}\right)=2$. Pick $h_{1}, h_{2} \in H\left(h_{1} \neq h_{2}\right)$ and consider vertices $u=\left(g_{1}, h_{1}\right), v=\left(g_{2}, h_{1}\right), z=\left(g_{2}, h_{2}\right) \in V(\Gamma)$. Note that, by the definition of $\Gamma, \partial_{\Gamma}(u, v)=\partial_{\Gamma}(u, z)=2$. Now count the number of common neighbours of vertices $u, v$ and $u, z$ in $\Gamma$. Note that

$$
N_{\Gamma}(u) \cap N_{\Gamma}(v)=\left\{(g, h) \mid g \in N_{G}\left(g_{1}\right) \cap N_{G}\left(g_{2}\right), h \in V(H) \backslash\left\{h_{1}\right\}\right\},
$$

and that

$$
N_{\Gamma}(u) \cap N_{\Gamma}(z)=\left\{(g, h) \mid g \in N_{G}\left(g_{1}\right) \cap N_{G}\left(g_{2}\right), h \in V(H) \backslash\left\{h_{1}, h_{2}\right\}\right\} .
$$


Therefore we have

$$
\left|N_{\Gamma}(u) \cap N_{\Gamma}(v)\right|=\left|N_{G}\left(g_{1}\right) \cap N_{G}\left(g_{2}\right)\right|(|V(H)|-1)
$$

and

$$
\left|N_{\Gamma}(u) \cap N_{\Gamma}(z)\right|=\left|N_{G}\left(g_{1}\right) \cap N_{G}\left(g_{2}\right)\right|(|V(H)|-2)
$$

Since $\Gamma$ is amply regular and $N_{G}\left(g_{1}\right) \cap N_{G}\left(g_{2}\right) \neq \emptyset$, it follows that $|V(H)|-1=|V(H)|-2$, a contradiction. Therefore, $G=K_{m}$ for some $m \geqslant 2$.

Theorem 22. $\Gamma=G[H]-n G$ is amply regular if and only if one of the following (i), (ii) holds:

(i) $H=E_{n}$ for some $n \geqslant 3$ and either $G=K_{2}$ or $G=K_{n}$.

(ii) $H=K_{n}$ for some $n \geqslant 3$ and $G=K_{m}$ for some $m \geqslant 2$.

Proof. Assume that $\Gamma$ is amply regular. By Lemma 17, $H=E_{n}$ or $H=K_{n}$ for some $n \geqslant 3$. By Lemma 21, $G=K_{m}$ for some $m \geqslant 2$. It remains to show that if $H=E_{n}$ and $G=K_{m}$ with $m \geqslant 3$, then $m=n$. Pick $g_{1}, g_{2} \in V(G)\left(g_{1} \neq g_{2}\right)$ and $h_{1}, h_{2} \in$ $V(H)\left(h_{1} \neq h_{2}\right)$. Consider vertices $u=\left(g_{1}, h_{1}\right), v=\left(g_{2}, h_{1}\right)$ and $z=\left(g_{1}, h_{2}\right)$ of $\Gamma$. Since $n, m \geqslant 3$ we have $\partial_{\Gamma}(u, v)=\partial_{\Gamma}(u, z)=2$. Furthermore, it is easy to see that $\left|N_{\Gamma}(u) \cap N_{\Gamma}(v)\right|=(m-2)(n-1)$ and that $\left|N_{\Gamma}(u) \cap N_{\Gamma}(z)\right|=(m-1)(n-2)$. It follows that $m=n$.

Conversly, assume that $H=E_{n}$ for some $n \geqslant 3$ and $G=K_{m}$ for $m \in\{2, n\}$. If $m=2$, then $\Gamma$ is isomorphic to $K_{n, n}-n K_{2}$, a complete bipartite graph minus 1-matching, which is clearly amply regular. If $m=n$, then it is easy to see that $\Gamma$ is amply regular with $\lambda_{\Gamma}=(n-2)^{2}$ and $\mu_{\Gamma}=(n-2)(n-1)$. Note that this graph is isomorphic to a complement of the $n \times n$ Rook's graph, the Cartesian product $K_{n} \square K_{n}$. Finally, if $H=K_{n}$ for some $n \geqslant 3$ and $G=K_{m}$ for some $m \geqslant 2$, then $\Gamma$ is isomorphic to the complete multipartite graph $K_{n \times m}$, which is clearly amply regular.

\section{Co-normal product}

Throughout this section let $G$ and $H$ be graphs and let $\Gamma$ be the co-normal product of $G$ and $H$. We will classify graphs $G$ and $H$ for which $\Gamma$ is connected edge regular graph. To avoid trivialities we assume that $|V(G)| \geqslant 2$ and $|V(H)| \geqslant 2$.

Lemma 23. $\Gamma$ is connected if and only one of the following holds:

(i) $H=E_{n}$ for some $n \geqslant 2$ and $G$ is connected.

(ii) $G=E_{n}$ for some $n \geqslant 2$ and $H$ is connected.

(iii) $G$ and $H$ are nonempty and at least one of $G$ or $H$ is without isolated vertices. 
Proof. Assume that one of (i), (ii), (iii) above holds and pick vertices $\left(g_{1}, h_{1}\right)$ and $\left(g_{2}, h_{2}\right)$ of $\Gamma$. We will show that $\Gamma$ is connected by displaying a walk between $\left(g_{1}, h_{1}\right)$ and $\left(g_{2}, h_{2}\right)$. Assume first that (i) above holds. If $g_{1}=g_{2}$ then pick a neighbour $g$ of $g_{1}$ in $G$, and observe that $\left(\left(g_{1}, h_{1}\right),\left(g, h_{2}\right),\left(g_{1}, h_{2}\right)\right)$ is a walk between $\left(g_{1}, h_{1}\right)$ and $\left(g_{2}, h_{2}\right)$. If $g_{1} \neq g_{2}$ then pick a path $\left(g_{1}, z_{1}, z_{2}, \ldots, z_{i}, g_{2}\right)$ between $g_{1}$ and $g_{2}$ in $G$. Note that in this case $\left(\left(g_{1}, h_{1}\right),\left(z_{1}, h_{1}\right),\left(z_{2}, h_{1}\right), \ldots,\left(z_{i}, h_{1}\right),\left(g_{2}, h_{2}\right)\right)$ is a walk between $\left(g_{1}, h_{1}\right)$ and $\left(g_{2}, h_{2}\right)$.

If (ii) above holds then clearly $\Gamma$ is connected since co-normal product is commutative.

Assume now that (iii) above holds. Since co-normal product is commutative, we could assume that $G$ is without isolated vertices. As $H$ is nonempty, it contains adjacent vertices $c, d$. Pick $z \in N_{G}\left(g_{1}\right)$ and $w \in N_{G}\left(g_{2}\right)$. Then $\left(\left(g_{1}, h_{1}\right),(z, c),(w, d),\left(g_{2}, h_{2}\right)\right)$ is a walk between $\left(g_{1}, h_{1}\right)$ and $\left(g_{2}, h_{2}\right)$.

We will now show that if $\Gamma$ is connected, then one of (i), (ii), (iii) above holds. If $H$ is empty, then clearly $G$ is connected. Indeed, if $\mathrm{G}$ is not connected, then pick $g_{1}, g_{2} \in V(G)$ which are in different components and pick $h \in V(H)$. Note that there is no path between $\left(g_{1}, h\right)$ and $\left(g_{2}, h\right)$, so $\Gamma$ is not connected, a contradiction. If $G$ is empty, then $H$ is connected from the commutativity of the co-normal product.

Finally, assume that none of $G, H$ is empty. Assume also that $g \in V(G)$ and $h \in V(H)$ are isolated vertices. Then $(g, h)$ is an isolated vertex of $\Gamma$, a contradiction. Therefore, at least one of $G, H$ is without isolated vertices, and so (iii) above holds.

Lemma 24. $\Gamma$ is regular if and only if both $G$ and $H$ are regular. In this case the valency $k_{\Gamma}=k_{G}|V(H)|+k_{H}|V(G)|-k_{G} k_{H}$.

Proof. Assume $\Gamma$ is regular graph of valency $k_{\Gamma}$. For an arbitrary vertex $g \in V(G)$ take vertices $\left(g, h_{1}\right),\left(g, h_{2}\right) \in V(\Gamma)$. By the definition of $\Gamma$ we have

$$
k_{\Gamma}=\left|N_{\Gamma}\left(\left(g, h_{1}\right)\right)\right|=\left|N_{G}(g)\right||V(H)|+\left|N_{H}\left(h_{1}\right)\right||V(G)|-\left|N_{G}(g)\right|\left|N_{H}\left(h_{1}\right)\right|,
$$

and

$$
k_{\Gamma}=\left|N_{\Gamma}\left(\left(g, h_{2}\right)\right)\right|=\left|N_{G}(g)\right||V(H)|+\left|N_{H}\left(h_{2}\right)\right||V(G)|-\left|N_{G}(g)\right|\left|N_{H}\left(h_{2}\right)\right| .
$$

It follows that $\left|N_{H}\left(h_{1}\right)\right|=\left|N_{H}\left(h_{2}\right)\right|$, so $H$ is regular. $G$ is regular for the commutativity of the co-normal product.

Conversely, if both $G$ and $H$ are regular, then $\Gamma$ is obviously a regular graph, as

$$
\left|N_{\Gamma}((g, h))\right|=\left|N_{G}(g)\right||V(H)|+\left|N_{H}(h)\right||V(G)|-\left|N_{G}(g)\right|\left|N_{H}(h)\right|,
$$

SO

$$
\left|N_{\Gamma}((g, h))\right|=k_{G}|V(H)|+k_{H}|V(G)|-k_{G} k_{H}
$$

for every $(g, h) \in V(\Gamma)$.

Lemma 25. Assume that $\Gamma$ is edge regular. Then the following (i), (ii) hold.

(i) Either $G=E_{m}$ for some positive integer $m$, or $G$ is edge regular. 
(ii) Either $H=E_{m}$ for some positive integer $m$, or $H$ is edge regular.

Proof. Recall that $G$ and $H$ are regular by Lemma 24 .

(i) Assume that $G$ is nonempty. Pick a pair of adjacent vertices $g_{1}, g_{2}$ of $G$. For an arbitrary vertex $h \in V(H)$ consider $u=\left(g_{1}, h\right), v=\left(g_{2}, h\right) \in V(\Gamma)$. Note that, by the definition of $\Gamma, \partial_{\Gamma}(u, v)=1$. We now count the number of common neighbours of $u$ and $v$. Observe that

$$
\begin{aligned}
N_{\Gamma}(u) \cap N_{\Gamma}(v)= & \left\{\left(g^{\prime}, h^{\prime}\right) \mid g^{\prime} \in N_{G}\left(g_{1}\right) \cap N_{G}\left(g_{2}\right), h^{\prime} \in V(H)\right\} \cup \\
& \left\{\left(g^{\prime}, h^{\prime}\right) \mid g^{\prime} \in V(G), h^{\prime} \in N_{H}(h)\right\} .
\end{aligned}
$$

Therefore

$\left|N_{\Gamma}(u) \cap N_{\Gamma}(v)\right|=\left|N_{G}\left(g_{1}\right) \cap N_{G}\left(g_{2}\right)\right||V(H)|+\left|N_{H}(h)\right||V(G)|-\left|N_{G}\left(g_{1}\right) \cap N_{G}\left(g_{2}\right)\right|\left|N_{H}(h)\right|$.

Since $\lambda_{\Gamma}=\left|N_{\Gamma}(u) \cap N_{\Gamma}(v)\right|$, it follows that

$$
\left|N_{G}\left(g_{1}\right) \cap N_{G}\left(g_{2}\right)\right|=\frac{\lambda_{\Gamma}-k_{H}|V(G)|}{|V(H)|-k_{H}} .
$$

Therefore, the number of common neighbours of two adjacent vertices of $G$ is independent of the choice of these vertices, and thus $\lambda_{G}$ exists. The result follows.

(ii) This follows from the commutativity of the co-normal product.

Corollary 26. If $\Gamma$ is edge regular and $G, H$ are nonempty, then

$$
\begin{aligned}
\lambda_{\Gamma} & =\lambda_{G}|V(H)|+k_{H}|V(G)|-\lambda_{G} k_{H} \\
& =\lambda_{H}|V(G)|+k_{G}|V(H)|-\lambda_{H} k_{G} \\
& =\lambda_{G}|V(H)|+2 k_{H}\left(k_{G}-\lambda_{G}\right)+\lambda_{H}\left(|V(G)|-2 k_{G}+\lambda_{G}\right) \\
& =\lambda_{H}|V(G)|+2 k_{G}\left(k_{H}-\lambda_{H}\right)+\lambda_{G}\left(|V(H)|-2 k_{H}+\lambda_{H}\right) .
\end{aligned}
$$

Proof. Observe that by Lemma 24 and Lemma 25, $G$ and $H$ are both edge regular, so $\lambda_{G}$ and $\lambda_{H}$ exist. The first equality now follows from (7). To prove the third equality, pick adjacent vertices $g_{1}, g_{2}$ of $G$ and adjacent vertices $h_{1}, h_{2}$ of $H$. Consider vertices $u=\left(g_{1}, h_{1}\right), v=\left(g_{2}, h_{2}\right) \in V(\Gamma)$. Note that, by the definition of $\Gamma, \partial_{\Gamma}(u, v)=1$. We now count the number of common neighbours of $u$ and $v$. Note that we have

$$
\begin{aligned}
N_{\Gamma}(u) \cap N_{\Gamma}(v)= & \left\{(g, h) \mid g \in N_{G}\left(g_{1}\right) \cap N_{G}\left(g_{2}\right), h \in V(H)\right\} \cup \\
& \left\{(g, h), \mid g \in N_{G}\left(g_{1}\right) \backslash N_{G}\left(g_{2}\right), h \in N_{H}\left(h_{2}\right)\right\} \cup \\
& \left\{(g, h), \mid g \in N_{G}\left(g_{2}\right) \backslash N_{G}\left(g_{1}\right), h \in N_{H}\left(h_{1}\right)\right\} \cup \\
& \left\{(g, h), \mid g \in V(G) \backslash\left(N_{G}\left(g_{1}\right) \cup N_{G}\left(g_{2}\right)\right), h \in N_{H}\left(h_{1}\right) \cap N_{H}\left(h_{2}\right)\right\} .
\end{aligned}
$$

Therefore

$$
\begin{aligned}
\left|N_{\Gamma}(u) \cap N_{\Gamma}(v)\right|= & \left|N_{G}\left(g_{1}\right) \cap N_{G}\left(g_{2}\right)\right||V(H)|+ \\
& \left(\left|N_{G}\left(g_{1}\right)\right|-\left|N_{G}\left(g_{1}\right) \cap N_{G}\left(g_{2}\right)\right|\right)\left|N_{H}\left(h_{2}\right)\right|+ \\
& \left(\left|N_{G}\left(g_{2}\right)\right|-\left|N_{G}\left(g_{1}\right) \cap N_{G}\left(g_{2}\right)\right|\right)\left|N_{H}\left(h_{1}\right)\right|+ \\
& \left(|V(G)|-\left|N_{G}\left(g_{1}\right) \cup N_{G}\left(g_{2}\right)\right|\right)\left|N_{H}\left(h_{1}\right) \cap N_{H}\left(h_{2}\right)\right| .
\end{aligned}
$$

THE ELECTRONic JOURNAL OF COMBINATORICS 20(1) (2013), \#P62 
The result follows.

The second and the fourth equality are obtained by reversing the roles of $G$ and $H$.

Theorem 27. $\Gamma$ is edge regular if and only if one of the following (i)-(iii) holds:

(i) $H=E_{m}$ for some $m \geqslant 2$ and $G$ is edge regular.

(ii) $G=E_{m}$ for some $m \geqslant 2$ and $H$ is edge regular.

(iii) $H=K_{s \times m}$ and $G=K_{t \times n}$ for some $s, t \geqslant 2$ and $m, n \geqslant 1$.

Proof. Assume that $\Gamma$ is edge regular. If $H=E_{m}\left(G=E_{m}\right.$, respectively) for some $m \geqslant 2$ then we are done, since $G$ ( $H$, respectively) is edge regular by Lemma 25. Assume now that $G, H$ are nonempty. By Corollary 26,

$$
\lambda_{G}|V(H)|+k_{H}|V(G)|-\lambda_{G} k_{H}=\lambda_{G}|V(H)|+2 k_{H}\left(k_{G}-\lambda_{G}\right)+\lambda_{H}\left(|V(G)|-2 k_{G}+\lambda_{G}\right) .
$$

It follows that

$$
k_{H}\left(|V(G)|-2 k_{G}+\lambda_{G}\right)=\lambda_{H}\left(|V(G)|-2 k_{G}+\lambda_{G}\right) .
$$

Since obviously $k_{H} \neq \lambda_{H}$ we have $2 k_{G}-\lambda_{G}=|V(G)|$. Note that in the complement of $G$, every two nonadjacent vertices have exactly $|V(G)|-2 k_{G}+\lambda_{G}=0$ common neighbours. Since the complement of $G$ is also regular, Proposition 1(ii) implies that the complement of $G$ is isomorphic to $t \times K_{n}$ for some $t, n \geqslant 1$. It follows that $G=K_{t \times n}$ for some $t, n \geqslant 1$. Since $G$ is nonempty, we also have that $t \geqslant 2$. The proof that $H=K_{s \times m}$ for some $s \geqslant 2$ and $m \geqslant 1$ follows from commutativity of the co-normal product.

Assume now that (i), (ii) or (iii) of the Theorem holds. Then $\Gamma$ is regular by Lemma 24 . It is now an easy exercise to show that if (i) ((ii), respectively) holds, then $\lambda_{\Gamma}=\lambda_{G}|V(H)|$ ( $\lambda_{\Gamma}=\lambda_{H}|V(G)|$, respectively), and so $\Gamma$ is edge regular. If (iii) holds, then $\Gamma=K_{s t \times m n}$, which is also edge regular.

Corollary 28. $\Gamma$ is amply regular if and only if one of the following (i)-(iii) holds:

(i) $H=E_{m}$ for some $m \geqslant 2$ and $G=K_{t \times n}$ for some $t \geqslant 2, n \geqslant 1$.

(ii) $G=E_{m}$ for some $m \geqslant 2$ and $H=K_{t \times n}$ for some $t \geqslant 2, n \geqslant 1$.

(iii) $H=K_{s \times m}$ and $G=K_{t \times n}$ for some $s, t \geqslant 2$, and either $m \geqslant 2$ or $n \geqslant 2$.

Proof. Assume that $\Gamma$ is amply regular. Let first consider case (i) of Theorem 27, that is the case when $H=E_{m}$ for some $m \geqslant 2$ and $G$ is edge regular. If $G$ is a complete graph, then (i) above holds. Therefore assume that $G$ is not a complete graph. Pick $g \in V(G)$ and $h_{1}, h_{2} \in V(H)$. Abbreviate $u=\left(g, h_{1}\right), v=\left(g, h_{2}\right)$ and note that $\partial_{\Gamma}(u, v)=2$. It is also clear that

$$
\left|N_{\Gamma}(u) \cap N_{\Gamma}(v)\right|=k_{G}|V(H)| .
$$

Next pick arbitrary $g_{1}, g_{2} \in V(G)$ with $\partial_{G}\left(g_{1}, g_{2}\right)=2$, and arbitrary $h \in V(H)$. Abbreviate $w=\left(g_{1}, h\right), z=\left(g_{2}, h\right)$ and note that $\partial_{\Gamma}(w, z)=2$. In this case we have

$$
\left|N_{\Gamma}(w) \cap N_{\Gamma}(z)\right|=\left|N_{G}\left(g_{1}\right) \cap N_{G}\left(g_{2}\right)\right||V(H)| .
$$


Since $\Gamma$ is amply regular, we clearly have

$$
k_{G}|V(H)|=\left|N_{G}\left(g_{1}\right) \cap N_{G}\left(g_{2}\right)\right||V(H)|,
$$

which implies $k_{G}=\left|N_{G}\left(g_{1}\right) \cap N_{G}\left(g_{2}\right)\right|$. Therefore, $\mu_{G}$ exists and is equal to $k_{G}$. Note that this implies that for $x, y \in V(G)$ we have $\partial_{G}(x, y) \leqslant 2$ (since in $G$ there is no induced path of length 3), and so by Proposition 1(iii), $G$ is $K_{t \times n}$ for some positive integers $t, n$. As $G$ is connected, we clearly have that $t \geqslant 2$.

Part (ii) follows from the commutativity of the co-normal product.

Let us now consider case (iii) of Theorem 27, that is $H=K_{s \times m}$ and $G=K_{t \times n}$ for some $s, t \geqslant 2$ and $m, n \geqslant 1$. Since $\Gamma$ is not a complete graph, we have either $m \geqslant 2$ or $n \geqslant 2$.

Conversely, if (i) or (ii) above holds, then $\Gamma=K_{t \times m n}$, and if (iii) above holds, then $\Gamma=K_{s t \times m n}$. In both cases $\Gamma$ is an amply graph.

\section{References}

[1] S. Aggarwal, P. K. Jha, and M. Vikram. Distance Regularity in Direct-Product Graphs. Appl. Math. Letters 13(1):51-55, 2000.

[2] A. E. Brouwer, A. M. Cohen and A. Neumaier. Distance-regular graphs. SpringerVerlag, New York, 1998.

[3] W. Imrich, and S. Klavžar. Product graphs. Wiley-Interscience, New York, 2000.

[4] S. Y. Song. Products of distance-regular graphs. Util. Math. 29:173-175, 1986.

[5] D. Stevanović. Distance regularity of compositions of graphs. Appl. Math. Letters $17(3): 337-343,2004$. 